\title{
ORIGENS E DISTRIBUIÇÕES DO NERVO OBTURATÓRIO EM FETOS DE JAVALIS (Sus scrofa scrofa LINNAEU - 1758)
}

\author{
ORIGINS AND DISTRIBUTION OF THE OBTURATORIUS NERVE IN WILD BOARS \\ FETUSES (Sus scrofa scrofa LINNAEU - 1758)
}

\section{Andréa Regina Abrantes GOMES ${ }^{1}$; Frederico Ozanam CARNEIRO E SILVA ${ }^{2}$; Roanne Yasmin Gonçalves VASCONCELOS ${ }^{1}$; Lázaro Antônio SANTOS ${ }^{1}$; André Luiz Quagliatto SANTOS ${ }^{2}$}

1. Pós-graduação em Ciências Veterinárias, Faculdade de Medicina Veterinária e Zootecnia - FAMEV, Universidade Federal de Uberlândia - UFU, Uberlândia, MG, Brasil. andreagomes85@gmail.com; 2. Docente, Doutor, FAMEV - UFU, Uberlândia, MG, Brasil.

\begin{abstract}
RESUMO: A criação de javalis vem crescendo no Brasil devido à procura de um produto de qualidade e com propriedades nutricionais. Analisou-se a origem e distribuição dos nervos obturatórios em 19 fetos de javalis (Sus scrofa scrofa) visando fornecer dados morfológicos para anatomia comparativa e áreas afins. A fixação destes animais em solução aquosa de formaldeído a $10 \%$ ocorreu mediante diferentes pontos de injeções subcutâneas, intramusculares e intracavitárias, bem como por imersão dos referidos exemplares em recipientes com a mesma solução por um período mínimo de 48 horas. Foi observado que o referido nervo originou-se dos ramos ventrais de $\mathrm{L}_{4}$ a $\mathrm{L}_{6}$. Distribui-se para os músculos obturatório externo, grácil, adutor e pectíneo. Pode-se afirmar que tanto a origem como a distribuição do nervo obturatório em javalis se assemelha aos padrões evidenciados em suínos domésticos.
\end{abstract}

PALAVRAS-CHAVE: Neuroanatomia. Plexo lombossacral. Suidae. Inervação.

\section{INTRODUÇÃO}

Nos últimos anos, no Brasil, tem-se notado um incremento na criação de animais silvestres, com a organização de criatórios específicos, com finalidades que vão do manejo e reprodução a exploração econômica. O javali (Sus scrofa scrofa) é preferido pelos consumidores, devido às suas propriedades organolépticas (cor vermelha e sabor diferenciado) e nutricionais (baixas taxas de gordura e colesterol), porém somente o javali puro possui tais características (SILVA, 2007).

$\mathrm{Na}$ escala filogenética, o javali é um parente do suíno doméstico e muitos criadores realizam o cruzamento entre essas espécies visando incrementar índices zootécnicos (SARUBBI, 2004). A distocia na suinocultura, não é tão frequente, porém pode levar a lesões no aparelho locomotor como distensões musculares e/ou ruptura de nervos do plexo lombossacral (CHAGAS et al., 2006). Decorrente destes cruzamentos, não se sabe se os animais resultantes podem apresentar maior suscetibilidade a estas injurias.

Ademais, o número de vértebras lombares nas espécies domésticas é variável e influência na quantidade e disposição dos nervos espinhais lombares (MIHELIAE et al., 2004). O plexo lombossacral é resultado da união de raízes ventrais de nervos espinhais lombares e sacrais, sendo o obturatório um de seus constituintes mais caudais (GHOSHAL, 1986).
Ao analisar a constituição deste nervo, este pode ter a mesma origem tanto para suínos como para ruminantes. De ramos ventrais oriundos de $\mathrm{L}_{4}$, $\mathrm{L}_{5}$ e $\mathrm{L}_{6}$ segundo Nickel et al. (1981), Chagas et al. (2006) e Dyce et al. (2010); com contribuições de $\mathrm{L}_{7}$ e $S_{1}$ de acordo com Miheliae et al. (2004), Miranda et al. (2007) e Vasconcelos et al. (2009), e também de $\mathrm{L}_{3}$, Ghoshal (1986).

No tocante a sua distribuição, em suínos, o nervo obturatório envia ramos aos músculos grácil, pectíneo, adutor, obturador externo (GHOSHAL, 1986; SCHWARZE; SCHRÖDER, 1991; SISSON; GROSSMAN, 2004; KONIG; LIEBICH, 2007), e quadrado femoral, semimembranáceo e sartório segundo Chagas et al. (2006). Tanto em suínos como nos demais animais domésticos, esta musculatura está envolvida no processo de adução e sustentação do membro.

Já quanto sua trajetória, este nervo direciona-se para extremidade cranial do forame obturado, cruza a superfície ventral da articulação sacro ilíaca, atingindo o corpo do osso ílio. Segue pelo assoalho da pelve em direção a este orifício, através do qual atinge os músculos mediais da coxa. Neste ponto onde se contrapõe ao osso, ele fica vulnerável. (VASCONCELOS et al., 2009).

$\mathrm{Na}$ ocorrência de lesão do referido nervo, gera-se sinais de disfunção como incapacidade de adução da articulação do quadril. O membro afetado pode não sustentar efetivamente $\mathrm{o}$ peso $\mathrm{e}$ os membros pélvicos são mantidos abertos, tendendo a 
se deslizarem lateralmente. Nestes casos, há perigo de ruptura dos músculos adutores ou de luxações coxofemorais pélvicas ou sacrais (FRANDSON et al., 2011).

O conhecimento da localização e distribuição dos principais nervos é determinante no diagnóstico clínico de lesões ou ausência de sensibilidade. Desta forma, objetivou-se com esta pesquisa descrever as origens e distribuições do nervo obturatório em fetos de javalis fornecendo assim dados morfológicos para anatomia comparativa, bem como para áreas afins como clinica e cirurgia de animais silvestres.

\section{MATERIAL E MÉTODOS}

O presente experimento teve como base a dissecação do nervo obturatório de 19 fetos de javalis (Sus scrofa scrofa), natimortos, procedentes de doações do IBAMA (Instituto Brasileiro do Meio Ambiente e dos Recursos Naturais Renováveis) e do LAPAS (Laboratório de Pesquisas com Animais Silvestres) da Universidade Federal de Uberlândia.

A fixação destes animais em solução aquosa de formaldeído a $10 \%$ ocorreu mediante diferentes pontos de injeções subcutâneas, intramusculares e intracavitárias, bem como por imersão dos referidos exemplares em recipientes com a mesma solução por um período mínimo de 48 horas antes do início da dissecação.

A fim de visualizar as origens dos nervos obturatórios direito e esquerdo foi feita uma incisão horizontal na linha mediana ventral desde o terço final da cavidade torácica até a borda caudal da sínfise pelvina. A partir dela, outras quatro incisões verticais foram realizadas, duas em cada antímero, até alcançar a linha mediana dorsal. Afastaram-se cranialmente os órgãos internos, aorta abdominal e veia cava caudal. Por conseguinte, desarticulou a sínfise pélvica rebatendo-a.

Após a retirada dos músculos psoas maior e menor, visualizaram-se os ramos ventrais dos nervos espinhas lombares de ambos antímeros que dão origem aos nervos obturatórios direito $\mathrm{e}$ esquerdo. Por fim, analisaram-se as distribuições dos ramos musculares dos nervos obturatórios em seus respectivos antímeros, na região medial da coxa.

A documentação dos resultados foi realizada a partir de observações e fotografias das origens e distribuições dos nervos obturatórios. A nomenclatura adotada para descrição dos resultados foi de acordo com o Nomina Anatômica Veterinária (INTERNATIONAL COMMITTEE ON VETERINARY GROSS ANATOMICAL NOMENCLATURE, 2012). Os dados foram compilados em tabelas e expressos por fotos, sendo que a análise estatística foi de forma descritiva através de porcentagem simples.

\section{RESULTADOS}

Nos 19 exemplares de javalis, verificou-se a presença de seis vértebras lombares e a origem do nervo obturatório nos antímeros direito e esquerdo foi proveniente dos ramos ventrais dos nervos espinhais lombares quatro, cinco e seis, visto que em dois casos $(10,52 \%)$ não foi constatado contribuições de $\mathrm{L}_{6}$ (Figura 1).

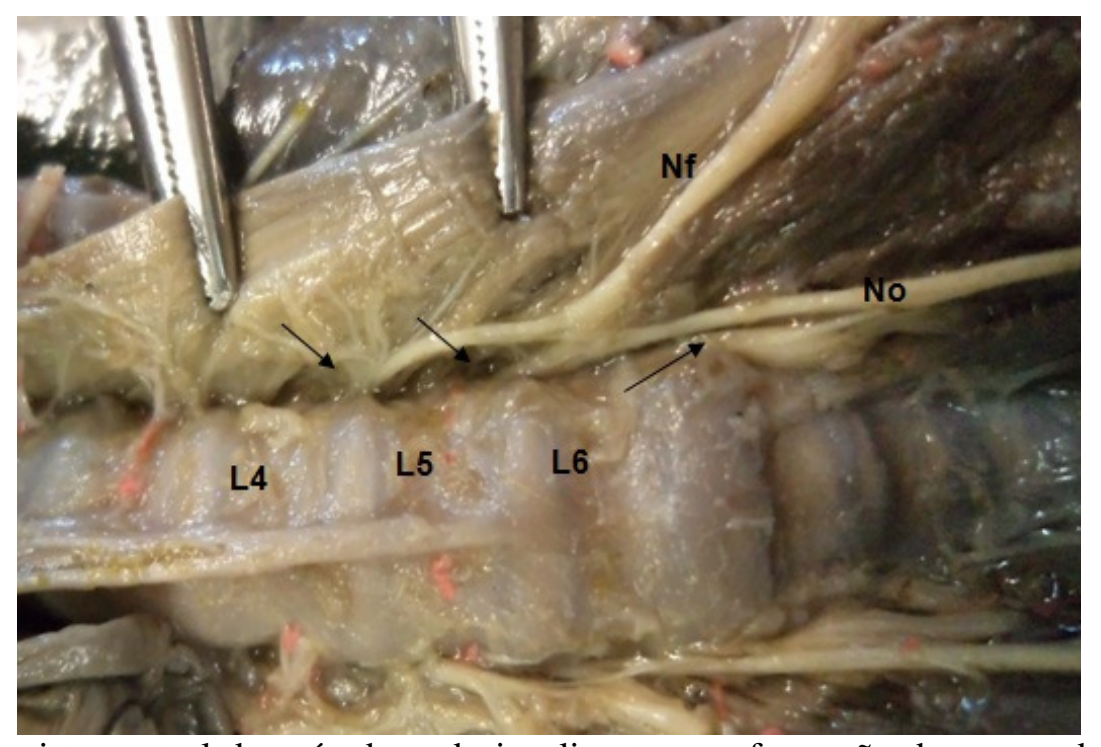

Figura 1. Fotografia da vista ventral das vértebras do javali nota-se a formação do nervo obturatório (No) a partir dos ramos ventrais (setas) dos nervos espinhais L4, L5 e L6. Observa-se, também, o nervo femoral (Nf). 
Ao longo de sua trajetória, o nervo obturatório é contínuo na face medial do corpo do ílio, em direção à borda cranial do forame obturatório. Emite ramos para o músculo obturatório externo antes de atravessar este orifício. Após, ramifica-se nos músculos grácil, adutor e pectíneo (Tabela 1 e Figura 2).

Tabela 1. Ramificações musculares do nervo obturatório nos antímeros direito (AD) e esquerdo (AE), quanto a frequência de animais por quantidade de ramo (\%) e total de animais (\%), nos 19 espécimes de fetos de javali (Sus scrofa scrofa), Uberlândia 2012.

\begin{tabular}{lcccc}
\hline \multirow{2}{*}{ Musculatura } & \multicolumn{2}{c}{ AD } & \multicolumn{2}{c}{ AE } \\
\cline { 2 - 5 } & Ramos $(\%$ animais $)$ & $\begin{array}{c}\text { Total de } \\
\text { animais } \%\end{array}$ & Ramos $(\%$ animais $)$ & $\begin{array}{c}\text { Total de } \\
\text { animais } \%\end{array}$ \\
\hline $\begin{array}{l}\text { Obturatório } \\
\text { externo }\end{array}$ & $\begin{array}{c}2(61,1) ; 3(33,3) ; \\
4(5,5)\end{array}$ & 94,73 & $\begin{array}{c}1(14,2) ; 2(42,8) ; \\
3(35,7) ; 4(14,2)\end{array}$ & 73,68 \\
Grácil & $1(6,2) ; 2(50) ; 3(43,5)$ & 84,21 & $1(21,4) ; 2(57,1) ;$ & 73,68 \\
& & & $3(21,4)$ & \\
Adutor & $1(7,6) ; 2(61,5) ;$ & 68,42 & $1(33,3) ; 2(50) ;$ & 63,15 \\
& $3(30,7)$ & & $3(16,6)$ & \\
Pectíneo & $1(75) ; 2(25)$ & 42,10 & $1(77,8) ; 2(22,2)$ & 47,36 \\
\hline
\end{tabular}

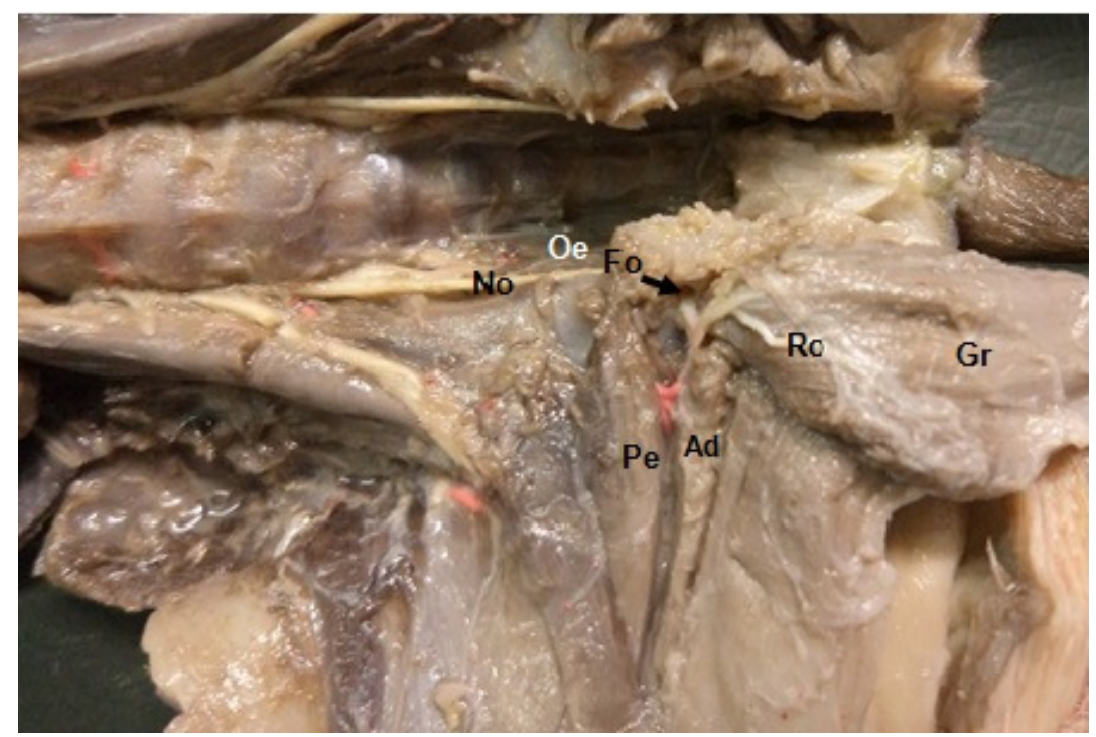

Figura 2. Fotografia da vista ventromedial da pelve e coxa do javali, evidencia-se o trajeto do nervo obturatório (No), emitindo ramos para o músculo obturatório externo (Oe) e atravessa o forame obturatório (Fo). Notam-se os músculos pectíneo (Pe), adutor (Ad) e grácil (Gr), o qual este último foi rebatido para visualizar um ramo $(\mathrm{Ro})$ do referido nervo.

\section{DISCUSSÃO}

Os resultados referentes à quantidade de vértebras lombares corroboram com os mencionados por Ghoshal (1986) e Miheliae et al. (2004) no qual o número, nas espécies domésticas, é variável, influenciando na quantidade de nervos espinhais lombares. Em javalis, identificou-se seis vértebras lombares em todos os espécimes, visto que esse número pode variar conforme espécie e raça (GHOSHAL, 1986).

Segundo Nickel et al. (1981), Chagas et al. (2006) e Dyce et al. (2010), o nervo obturatório é formado pela continuação dos ramos ventrais dos nervos espinhais lombares $\left(\mathrm{L}_{4}, \quad \mathrm{~L}_{5}\right.$ e $\left.\mathrm{L}_{6}\right)$, conformação observada em 90,48\% dos espécimes 
estudados. Entretanto, Cox et al. (1975), Sisson e Grossman (2004), Konig e Liebich (2007), não evidenciaram ramos de $\mathrm{L}_{4}$ tanto em suinos como em ruminantes, ao passo que, este apresenta pequena participação em cães, de acordo com Evans e Lahunta (1994).

Já Miheliae et al. (2004) e Vasconcelos et al. (2009) relataram a participação de $\mathrm{L}_{7}$, em suínos e ovinos respectivamente; e Miranda et al. (2007) evidenciaram a colaboração de ramos ventrais de $S_{1}$ em bovinos azebuados; porém, na presente investigação não foram notados tais ramos na constituição do nervo.

O nervo obturatório originou-se, em conjunto com o nervo femoral, em todos os casos analisados, o que esta em concordância com Ghoshal (1986) e Dyce et al. (2010), os quais afirmaram que, em suínos e ruminantes, pode ter a mesma origem do nervo femoral $\mathrm{L}_{4}, \mathrm{~L}_{5}$ e $\mathrm{L}_{6}$.

Em javalis (Sus scrofa scrofa), o nervo obturatório após originar-se entre $\mathrm{L}_{4}$ a $\mathrm{L}_{6}$, segue caudoventralmente ao longo do corpo do osso ílio, como foi observado em suínos por Chagas et al. (2006), em ruminantes por Miranda et al. (2007) e Vasconcelos et al. (2009) e carnívoros, por Evans e Lahunta (1994).

De acordo com Schwarze e Schröder (1991) e Dyce et al. (2010), o nervo acompanha paralelamente o tendão do músculo psoas menor e, antes de alcançar o forame obturador, emite ramos ao músculo obturador interno, fato não constatado nesta investigação.

Como evidenciado em suínos (NICKEL et al., 1981; GHOSHAL, 1986; SCHWARZE; SCHRODER, 1991; SISSON; GROSSMAN, 2004; CHAGAS et al., 2006; KONIG; LIEBICH, 2007; DYCE et al., 2010), ruminantes (COX et al., 1975; MIRANDA et al., 2007; VASCONCELOS et al.,
2009) e caninos (EVANS; LAHUNTA, 1994), o nervo obturatório em javalis após emergir do forame obturador emitiu ramos ao músculo pectíneo, adutor e grácil. Todavia, Chagas et al. (2006) identificaram, em suínos da linhagem AG 1050, ramos para os músculos sartório, quadrado femoral e semimembranáceo, o qual não foi notado neste estudo.

Devido ao trajeto do nervo obturatório, observou-se sua vulnerabilidade às injurias, o que faz com que os músculos adutores da coxa sejam susceptíveis a traumatismos. Segundo Chagas et al. (2006) e Frandson et al. (2011), a lesão do nervo obturatório na região pélvica ocasiona dificuldade de locomoção ou de permanecer em pé. Decorrente da proximidade com o canal do parto, esse nervo pode sofrer lesões durante a expulsão de feto.

\section{CONCLUSÕES}

Os nervos obturatórios em fetos de javalis originaram-se dos ramos ventrais de $\mathrm{L}_{4}$ a $\mathrm{L}_{6}$, em conjunto com os nervos femoral e isquiático, distribuíram-se nos músculos obturatório externo, grácil, pectíneo e adutor.

Dentre os animais domésticos comparados, pode-se concluir que tanto a origem como a distribuição do nervo obturatório em fetos de javalis se assemelham as evidenciadas nos suínos domésticos, exceto pela ausência de inervação dos músculos sartório, quadrado femoral e semimembranáceo, os quais estão presentes nestes últimos.

\section{AGRADECIMENTOS}

Ao IBAMA e LAPAS por disponibilizarem as peças.

\begin{abstract}
The creation of wild boars is increasing in Brazil due to demand for a quality product and excellent nutritional properties. This study analyzed the origin and distribution of the obturatorius nerve in 19 fetuses of wild boar (Sus scrofa scrofa) in order to provide morphological data for comparative anatomy and areas related. The introduction of these animals in aqueous formaldehyde $10 \%$ was by different points of subcutaneous, intramuscular and intracavitary injections, as well as by immersion of those specimens, in containers of the same solution for a minimum of 48 hours. It was noted that the nerve was originated from the ventral branches of $\mathrm{L}_{4}, \mathrm{~L}_{5}$ and $\mathrm{L}_{6}$. Concerning to distribution, the nerve dispatched ventral branches to the external obturator, gracilis, adductor and pectineus muscles. It can be stated that both the origin and distribution of the obturatorius nerve in wild boars is similar to the patterns evidenced in domestic swine.
\end{abstract}

KEYWORDS: Neuroanatomy. Lumbosacral plexus. Suidae. Innervation. 


\section{REFERÊNCIAS}

CHAGAS, R. G.; DRUMMOND, S. S.; SILVA, F. O. C.; EURIDES, D.; ALVES, E. C. M.; MIRANDA, R. L. Origem e distribuição do nervo obturatório em suínos (Sus scrofa domesticus - Linnaeus, 1758) da linhagem AG-1050. Arquivo de Ciência Veterinária e Zoologia, Umuarama, v. 9, n. 1, p. 15-20, 2006.

COX, N. S.; BREAZILE, J. E.; HOOVER, T. R. Surgical and anatomical study of calving paralysis. American Journal of Veterinary Research, Missouri, v. 36, n. 4, p. 427-430, 1975.

DYCE, K. M.; SACK, W. O.; WENSING, C. J. G. Tratado de anatomia veterinária. 4. ed. Rio de Janeiro: Guanabara Koogan, 2010. 834p.

EVANS, H.; LAHUNTA, A. Abdome, pelve e membro pélvico. In: EVANS, H.; LAHUNTA, A. Guia para a dissecação do cão. 3. ed. Rio de Janeiro: Guanabara Koogan, 1994. p.100-151.

FRANDSON, R. D.; WILKE, W. L.; FAILS, A. D. Anatomia e fisiologia dos animais da fazenda. 7a . ed. Rio de Janeiro: Guanabara Koogan, 2011. 432p.

GHOSHAL, N. G. Nervo espinhais: suíno. In: GETTY, R. Sisson/Grossman anatomia dos animais domésticos. 5a . ed. Rio de Janeiro: Guanabara Koogan, 1986, v. 2, p. 1294-1307.

INTERNATIONAL COMMITTEE ON VETERINARY GROSS ANATOMICAL NOMENCLATURE. Nomina Anatômica Veterinária. 15. ed. (edição revisada). Columbia: Committee Hannover, 2012. 177p.

KONIG, H. E.; LIEBICH, H. G. Veterinary anatomy of domestic mammals: textbook and colour atlas. 3. ed. Germany: Schattauer, 2007. 771p.

MIHELIAE, D.; GJURÈEIAE-KANTURAL, V.; MARKOVINOVIAE, S.; DAMJANOVIAE, A.; TRBOJEVIAE-VUKIÈEVIAE, T. Variations of formation of $\mathrm{n}$. femoralis, $\mathrm{n}$. obturatorius and $\mathrm{n}$. ischiadicus in pigs. Veterinarski Arhiv, Croacia, v. 74, n. 4, p. 261-270, 2004.

MIRANDA, R. L.; SILVA, F. O. C.; SEVERINO, R. S.; DRUMMOND, S. S.; GONÇALVEZ, R. C. Origens e distribuições dos nervos obturatórios em fetos fêmeas de bovinos azebuados. Bioscience Journal, Uberlândia, v. 23 , n. 4 , p. 120-127, 2007.

NICKEL, R.; SCHUMMER, A.; SEIFERLE, E. The anatomy of the domestic animals. v. 3. Berlin: Verlag Paul Parey, 1981. 610p.

SARUBBI, J. Javali: Qualquer semelhança com o porco doméstico não é mera coincidência. Associação Paulista dos Criadores de Suínos - APCS. Dicas Técnicas. 2004. Disponível em: < http://www.apcs.com.br/7,1,26,6837 > Acesso em 8 de julho de 2012.

SCHWARZE, E.; SCHRÖDER, L. Compendio de anatomia veterinária. 9. ed. Zaragoza: Acribia, 1991. 350 p.

SILVA, P. V. C. Caracterização genética de javalis por meio de microssatélites. 2007. 46f. Dissertação (Mestrado em Genética e Melhoramento Animal) - Faculdade de Ciências Agrárias e Veterinárias, Universidade Estadual Paulista “Julio de Mesquita Filho", Jaboticabal, 2007.

SISSON, S.; GROSSMAN, J. D. Neurologia. In: GETTY, R. Anatomia de los animales domésticos. 5. ed. Barcelona: Masson, 2004. 1046p.

VASCONCELOS, B. G.; SILVA, F. O. C.; SENA, C. V. B.; BITTAR, W. A.; VASCONCELOS, R. Y. G.; RESENDE, G. G. N.; RINALDI, F. C. Q.; SANTOS, M. C. D. Origens e distribuições dos nervos obturatórios em ovinos sem raça definida. Veterinária Notícias, Uberlândia, v. 15, n. 1, p. 41-46, 2009. 